\title{
PARA LEER LA FILOSOFÍA DEL DERECHO DE HEGEL*
}

\section{Julio De Zan ${ }^{* *}$}

1.

En el primer parágrafo de la Filosofía del Derecho enuncia Hegel la definición, escueta y precisa, del objeto de esta parte de la filosofía: "La ciencia filosófica del derecho tiene por objeto la idea del derecho, esto es, el concepto del derecho y su realización" (Rph $\ 1)^{1}$. Al final de la "Introducción" define a su vez el derecho como "la existencia de la voluntad libre (Dasein des freien Willens)" (\$29). La Primera Parte está dedicada, como sabemos, al derecho abstracto, y comienza con la deducción del concepto de persona a partir de la libertad individual de un sujeto $(\$ 34)$. La persona es el sujeto de los derechos que estudiará en toda esta Primera Parte, comenzando por la propiedad privada. Con este comienzo adopta la obra, por lo menos en su disposición externa, la forma de un tratamiento típicamente moderno, conforme al planteamiento del individualismo metodológico de las teorías jusnaturalistas, o liberales, en el sentido del debate actual entre el republicanismo y el liberalismo.

* El texto que aquí presento es una nueva versión de algunos pasajes del Cap. 13, "Derecho, moralidad y eticidad", de mi libro La Filosofía social y politica de Hegel, Buenos Aires, Ediciones del Signo, 2009.

** Investigador Principal del CONICET. Profesor Titular de la Universidad Nacional de General San Martín. Dirección electrónica: jdezan@arnet.com.ar

${ }^{1} \mathrm{La}$ abreviatura corresponde al título alemán Grundlinien der Pbilosophie des Rechts, que se simplifica como: Rechtsphilosophie. Las referencias de las citas de la obra se consignan en el cuerpo del texto, con la abreviatura Rph., o directamente con la indicación del número de parágrafo. La primera edición publicada por Hegel lleva como pie de imprenta los datos siguientes: Berlin, 1821. In der Nicolaischen Buchhandlung. Ahora sabemos que el libro había aparecido ya en octubre de 1820 . Por esta razón se la cita a veces con esta última fecha. Sobre las vicisitudes de la. publicación del libro, cfr. H. Christian Lucas / U. Rameil. "Furcht vor der Zensur? Zur Entstehung -und Durckgeschichte von Hegels Grundlinien der Philosophie des Rechts", en Hegel-Studien, Bd. 15, 1980, pp. 61-80. 
Este es el aspecto que ha enfatizado en su interpretación M. Riedel:

No obstante que Hegel se distancia expresamente de los extremos teoréticos de Hobbes, Rousseau y Kant, la entera disposición de la Filosofia del Derecho permanece fiel, en un aspecto esencial, al punto de vista jusnaturalista: su desarrollo conceptual comienza con la voluntad singular de un sujeto en su relación a las cosas de la naturaleza (propiedad) y en su relación con la voluntad de los otros individuos (contrato), es decir, comienza con el derecho que, en cuanto derecho abstracto, reconstruye la situación prepolítica de la doctrina del derecho natural. El movimiento que parte de la voluntad individual atraviesa todo el sistema del derecho -lo cual ha sido frecuentemente pasado por alto-, hasta la deducción incluso de la voluntad que se concentra en el Estado, la cual debe ser, asimismo, según Hegel, una voluntad individual (la voluntad del monarca) ${ }^{2}$.

Habrá que preguntarse por lo tanto si con este plan de su exposición se está retractando Hegel de su fuerte crítica a las teorías del derecho natural en su artículo de 1802, y si habría que leer el texto entonces en clave jusnaturalista. En este sentido, para Henning Ottmann el comienzo de la Filosofía del Derecho con figuras abstractas de las cuales no se puede deducir nada concreto y verdaderamente real, es una hipoteca a partir de la cual no es posible ningún progreso inmanente del concepto en sentido propiamente hegeliano ${ }^{3}$.

2 M. Riedel, "Natur und Freiheit in Hegels Rechtsphilosophie", en el libro del mismo autor: Materialien $z^{u}$ Hegels Rechtsphilosopbie, vol. II, Frankfurt, Suhrkamp, 1975, p. 117.

3 "Der Anfang der Rechtsphilosophie bei Sphären, denen in ihrer Konstrukthaftigkeit keine Bestandsfähigkeit und Schlüssigkeit entspricht, belastet die Rechtsphilosophie mit einem Beginn, aus dem sich kein immanentes Fortschreiten mehr erzeugen kann. Statt vom Abstrakten zum Konkreten führt der Weg von vorausgeschickten Abstraktionen eines Konkretes $\mathrm{zu}$ dem immer schon vorauszusetzenden Konkreten zurück - vom "Schein" Konkretion hervorbringender Abstraktion zur Wahrheit jenes Konkreten, in welchem das Abstrakte bereits gegründet ist" (Henning Ottmann, "Hegels Logik und Rechtsphilosophie. Unzulängliche Bemerkungen zu einem ungelösten Problem", en D. Henrich/R.-P. Horstmann, Hegels Philosophie des Rechts, Stuttgart, Klett-Cotta, 1982, pp. 385-386. 
Es cierto que para Hegel el sentido de los derechos y de la moralidad autónoma son presupuestos conceptuales necesarios para la comprensión de la eticidad moderna, y constituyen las condiciones normativas de las instituciones y del Estado en esta época, en cuanto diferentes de la eticidad sustancial inmediata del mundo antiguo. "Pero -como observa $\mathrm{H}$. Ottmannsi el derecho y la moralidad son condiciones necesarias de una eticidad desarrollada, solamente la eticidad parece ser, a su vez, la condición suficiente que garantiza la existencia y la permanencia del derecho y de la moralidad"4. El propio Hegel va a enfatizar además que el plan de la exposición, cuya Primera Parte trata del "derecho abstracto" de la persona, conjuntamente con el tratamiento de la moralidad, que le sigue inmediatamente en la Segunda Parte, antes de la exposición del sistema de las instituciones del mundo ético, no refleja ni el orden genético, o temporal, ni la estructura ontológica de la cosa misma. Para evitar malentendidos se requiere por lo tanto un trabajo hermenéutico que ha dado lugar a diferentes interpretaciones sobre el método y la estructura general de esta obra. Ia pregunta es, entonces, ¿a qué criterios obedece esta problemática estructura de la Filosofia del derecho? ¿Se trata quizás del efecto de la trasposición didáctica de los contenidos (dado que el texto está pensado como un manual "para guía de los oyentes de las Lecciones" del autor), o de una concesión inicial a la forma de los tratados clásicos de la época? ¿Puede haber otra razón de la disposición que presenta la materia, aparentemente extraña al sistema?

Si leemos la "Introducción" a la Tercera Parte sobre "La Eticidad", es claro que el punto de vista de Hegel es el de la sustancialidad del murido ético, que se expone en esta última Parte, y que el verdadero comienzo filosófico sistemático está recién allí. En el comentario de las Lecciones al $\S$ 156 el propio Hegel parece criticar incluso como no filosófico el plan de la exposición que él mismo ha seguido en las dos partes anteriores: "En el campo de lo ético son posibles siempre solamente dos puntos de vista: $\mathrm{o}$ bien se parte de la sustancialidad, o se procede de manera atomística elevándose a partir de los individuos. Este último punto de vista carece de espíritu...". Podría decirse que el planteamiento de Hegel revela ya

${ }^{4}$ Ibid., p. 383. 
"propósitos cruzados" en el sentido en que Charles Taylor emplea esta expresión con referencia al debate liberal-comunitario ${ }^{5}$.

\section{2.}

La primera pista para orientarse en la lectura de la obra está ya en la formulación del citado parágrafo primero de la Filosofía del Derecho, según el cual el objeto de esta ciencia no es el mero concepto, como en el jusnaturalismo, sino la idea del derecho y su realización. La idea del derecho no es ninguna abstracción, sino que es la realidad misma de la libertad concreta, la libertad realizada, en cuanto concepto existente que se ha dado una configuración, o que ha configurado el mundo conforme a su concepto. Esta configuración, que no existe solamente como concepto, es el otro momento esencial de la idea, el momento de su realidad, que es el punto de partida para la comprensión del concepto de la cosa.

La filosofía trata con ideas, y no con lo que puede llamarse meros conceptos. Ella muestra, por el contrario, la unilateralidad y falta de verdad de estos últimos, así como que el concepto (no lo que con frecuencia recibe este nombre, pero no es más que la determinación abstracta del entendimiento) es lo único que posee realidad, precisamente porque él se la da a sí mismo $(\$ 1, O b s$.$) .$

La citada declaración vuelve a dejar planteado sin embargo el problema inicial de manera aún más aguda, porque el tratamiento del derecho abstracto y de la moralidad parece trabajar precisamente con meros conceptos y definiciones a priori, o deducidos no se sabe de dónde. Para leer estos textos hay que despejar ante todo esta apariencia, porque lo que Hegel hace es lo que ya había enunciado en el "Prólogo" y eni el $\ 3$, a saber, analizar y reconstruir críticamente la racionalidad inmanente a los conceptos existentes del derecho y de la moralidad. En tal sentido el punto de partida de Hegel está en la experiencia histórica y los conceptos del derecho

5 Ch. Taylor, "Propósitos cruzados: el debate liberal-comunitario", en N. L. Rosenblum, El liberalismo y la vida moral, Buenos Aires, Nueva Visión, 1993, pp. 177 y ss. 
vigente, porque ésta es la fuente del conocimiento, tanto de la ciencia positiva del derecho como de la filosofía.

La exposición no es entonces, por cierto, meramente empíricodescriptiva, sino que los fenómenos histórico-sociales y políticos de su tiempo son expuestos y analizados como ya pensados en los conceptos de la Ciencia jurídica, de la Staatswissenschaft y de la Filosofía. Se trata de comprender el sentido y la racionalidad de las cosas a partir de conceptos, y de confrontar al mismo tiempo estos conceptos con la realidad. Hegel pone en obra ya de este modo el método de la "Teoría crítica de la sociedad", que será expuesto por la Escuela de Frankfurt como tematización reflexiva sobre los procedimientos reconstructivos de la obra de Marx, sin reconocer de manera adecuada en este aspecto la deuda de este último con $\mathrm{Hegel}^{6}$. Este tratamiento de la materia del espíritu objetivo, y el concepto mismo de la crítica interna, solamente se pueden comprender a partir de una actitud frente a la realidad que presupone la confianza hermenéutica de que hay un sentido por descubrir en las cosas del mundo de la cultura y de la historia, la cual se expresa en la famosa fórmula según la cual "todo lo real es racional". De la misma manera que la figura deforme, o el comportamiento irracional de un individuo, no deben ser obstáculo para ver que se rrata de un hombre, lo mismo ocurre, dice Hegel, con las realizaciones históricas de las ideas éticas, como el Estado. Pero el punto de vista hermenéutico de Hegel es ya el de una hermenéutica racional, que somete al análisis crítico las formas empíricas y las pretensiones de validez de las conceptualizaciones del pensamiento a partir de la idea esencial inmanente a la cosa misma. Es preciso leer entonces el texto como una teoría critica de la sociedad y del Estado moderno. La crítica de la crítica, que Hegel reitera especialmente desde la Fenomenología del Espíritu, se refiere a la crítica del conocimiento en el sentido de Kant, o a la crítica externa y abstracta de los sistemas filosóficos. Pero él construye su propio pensamiento al hilo de la exposición y la crítica interna (Darstellung und Kritik) de la comprensión del mundo de sus antecesores y de

${ }^{6}$ La claridad analítica de la exposición de Raymond Geuss en su libro: The Idea of a Critical Theory. Habermas and the Frankfurt School, Cambridge/New York/Melbourne, Cambridge University Press, 1981, esp. Cap. III, permite ver de manera especial ia filiación hegeliana de la idea de teoría crítica, aunque este autor tampoco la tiene en cuenta. 
sus contemporáneos, en tanto en ella se reflejan las contradicciones de su época. Esto se puede comprobar ya desde sus primeras publicaciones del período de Jena ${ }^{7}$. El ensayo de 1802/03 sobre las maneras de tratar científicamente el derecho natural está dedicado a la crítica de los principios de las teorías jusnaturalistas, mostrando cómo en las modernas teorías sobre esta materia se puede "ver reflejada la situación empírica del mundo en el espejo ideal de la ciencia", la cual solidifica y consolida sus contradicciones. En este ensayo temprano se plantea ya Hegel el problema que reencontramos en las dos primeras partes de Filosofía del Derecho. Dado que en este ensayo se trata ante todo de la crítica, lo propiamente positivo de la Filosofía de la eticidad no tendrá aquí su adecuado desarrollo, advierte el autor, sino que opera como un presupuesto que se explicita a través de la exposición y de la crítica interna de las teorías tradicionales ${ }^{8}$.

Las determinaciones conceptuales de la exposición no son por lo tanto ninguna construcción apriorística, ni se pueden comprender como generadas deductivamente a partir de las partes precedentes del sistema. Lo que hace Hegel no es aplicar las formas lógicas al material empírico, sino que es más bien la reconstrucción hermenéutica racional de los presupuestos de las concepciones del derecho, de la moralidad, de la sociedad civil, de lo político y del Estado, tal como aparecen constituidos en la época moderna y en su Filosofía, y explicitar, con ayuda de la lógica, sus determinaciones constitutivas, esenciales y necesarias, separando lo que no se corresponde, o se contradice con el concepto de la cosa. En cuanto a la Ciencia de la Lógica, a la que se remite constantemente el filósofo, hay que decir que la lógica solamente da sentido en cuanto es recordada como el pasado esencial de la Filosofía Real?. Pero la materia y el punto de partida

${ }^{7}$ Cfr. los artículos del Diario Crítico de Filosofía (Jena 1801-18003): "Sobre la esencia de la Filosofía crítica en general y especialmente sobre su relación con el estado actual de la Filosofía" (1801); "La relación del escepticismo con la Filosofía. Exposición de sus diferentes modificaciones y comparación del nuevo escepticismo con el antiguo" (1802); también el ensayo sobre creer y saber (1802), cuyo tema central es la crítica de lo que él llama "la filosofía de la reflexión de la subjetividad".

8 Jenaer Schriften, en G.W.F Hegel, Werke II, Frankfurt, Suhrkamp Verlag, 1970.

${ }^{9}$ Cfr. F. Duque, "El desarrollo del derecho abstracto y su lógica", en Taula. Quaderns de pensament, núm. 17-18, 1992, p. 66. 
están dados por la realidad práctica concreta de estas distintas esferas en su evolución histórica y en su realización en cuanto objetivadas en el mundo ético de la sociedad moderna y de sus instituciones.

Como lo declara el filósofo en las famosas expresiones del "Prólogo", que él mismo aplica metódicamente, especialmente aquí, tanto en su propia exposición como en la manera de tratar las otras concepciones filosóficas con las que discute:

la tarea de la filosofía es concebir lo que es, porque lo que es la razón. En lo que respecta al individuo, cada uno es, sin más, hijo de su tiempo; y así también la filosofía es su tiempo aprehendido en pensamientos... En cuanto pensamiento del mundo, la filosofía aparece en el tiempo después de que la realidad ha consumado el proceso de su formación y se ha preparado [para ello]. Lo que enseña el concepto es [por lo tanto], lo que muestra [ya] del mismo modo la historia como necesario; solamente cuando la realidad ha madurado aparece lo ideal frente a lo real y erige para sí el mismo mundo, aprehendido en su sustancia, en la figura de un reino intelectual ${ }^{10}$.

Si bien este pensamiento vale para el concepto de la filosofía en general, no es casual por cierto que aparezca formulado de la manera más contundente precisamente en el "Prólogo" de la Filosofía del Derecho. Para comprender el significado concreto de la obra, hay que leerla de la misma manera como ha sido escrita. Si el lector pretende ser más hegeliano, o más cerradamente sistemático que el propio Hegel, se perderá en un razonar abstracto, Hegels inmanent, y en la búsqueda de las correspondencias de cada paso con los pasajes de la Ciencia de la lógica, sin comprender su significado histórico. En este contexto identifica incluso Hegel expresamente "la verdadera comprensión histórica" con "el auténtico punto de vista filosófico" (die wabrhafte bistorische Ansicht, den echt philosophischen Standpunkt) ${ }^{11}$.

${ }^{10}$ G.W.F. Hegel, Werke, ed. cit., VII, p. 26 y 28.

${ }^{11}$ Ibid., VII, p. 35. 


\section{3.}

A renglón seguido de la notable afirmación citada al final del punto anterior, comienza sin embargo una larga y dura polémica contra el procedimiento de la explicación puramente histórica del origen de las instituciones, en la medida en que se pretende utilizarla como método para la justificación de su validez. La polémica se personaliza en la discusión del pensamiento de una de las autoridades de la ciencia jurídica, Gustav Hugo, pero el texto puede considerarse en general como una refutación del historicismo (y del positivismo jurídico), y más ampliamente aún, del método genealógico en tanto confunde la explicación genética con la justificación (o la crítica) de la valide $z^{12}$.

El considerar el surgimiento y el desarrollo de las determinaciones jurídicas, tal como se manifiestan en el tiempo (in der Zeit erscheinende), esa preocupación meramente bistórica... no guarda relación alguna con la consideración filosófica, dado que, en efecto, el desarrollo a partir de causas históricas no puede confundirse con el desarrollo a partir del concepto, y la explicación y justificación histórica (die geschicbtliche Erklärung und Rechtfertigung) no tienen el alcance y el significado de una justificación válida en síy por si’'13,

Es decir, el punto de vista de Hegel se sitúa a igual distancia del racionalismo jusnaturalista y del historicismo de la Escuela histórica del derecho.

El plan de la exposición de la Filosofía del Derecho invierte el orden del tiempo en la formación histórica del Derecho, la Moralidad y la Eticidad, secuencia que el propio Hegel ha enunciado con ciaridad en diferentes

${ }^{12}$ Como escribe L. Siep en su estudio sobre el "Prologo" de ia Filosofia del Derecho, "Cuando Hegel en los pasajes polémicos de la obra ataca sobre todo a Fries y a Hugo, apunta con toda probabilidad también a sus más importantes e influyentes colegas berlineses, a Schleiermacher (por sobre el primero), y a Savigny (detras del segundo)" L. Siep, en: Hegel, Grundlinien der Philosophie des Rechts, Berlin, Akademie Verlag. 1997, pp. 5-30.

${ }^{13}$ G.W.F. IWerke, VII, 35. 
textos $^{14}$. En la Eticidad antigua de las comunidades premodernas la moralidad y el derecho se encontraban como fundidas, o formando una unidad simbiótica, y no diferenciadas incluso de la religión. La autonomización de estos ámbitos de la vida del espíritu es un fenómeno tardío de la modernidad ${ }^{15}$. El tratamiento de los puntos de vista del derecho y de la moralidad que se han desarrollado en la época moderna, con los que comienza la Filosofía del Derecho, cobra en la exposición hegeliana, como lo ha destacado recientemente L. Siep, la forma de "una ciencia conceptual que no deduce sus contenidos de unos primeros principios, sino que explicita de manera reflexiva las presuposiciones del pensamiento que la cultura jurídica y política europea ha producido en la madurez de su historia..."16. Lo mismo puede decirse de la cultura moral de la modernidad, que se ha hecho reflexiva con Kant.

Las determinaciones [del concepto] en su existencia forman una serie de configuraciones [históricas] (eine Reibe von Gestaltungen)... es bajo esta forma de su existencia que son para nosotros conocidas las configuraciones. Porque nosotros sabemos lo que es la familia, la sociedad civil, etc., es decir, tenemos todo esto en la representación (in der Vorstellung)... [Pero] el punto de partida no es tampoco la mera representación, sino la elaboración de esta por el corpus jurídico de la

${ }^{14}$ Cfr. J. De Zan, op. cit., cap.13.

${ }^{15}$ En su tratado sistemático de Filosofía política y del Derecho (Facticidad y validez (1992), J. Habermas parte de la misma concepción, aunque no cita a Hegel, quizás porque tiene en la mente el orden de la exposición de la Rechtsphilosophie. Este autor realiza un significativo aporte, mediante dos principios formulados en el contexto teórico de la Filosofía contemporánea, pero que están en la línea de la concepción hegeliana de la superación, tanto del normativismo jusnaturalista de la Filosofía política fundada en los derechos, como del historicismo y del empirismo de la real politik, que privan a la política de toda dimensión normativa, y permiten pensar la autonomía de la política frente a la moral, manteniendo abierta al mismo tiempo la necesaria conexión entre ambas. Este resultado lo obtiene Habermas a través del Principio del Discurso como el meta-principio epistémico de toda la Filosofía práctica, anterior a la diferenciación de la Ética, la política y el Derecho, y del principio de la cooriginariedad de los derechos y de la soberanía política, que no se derivan el uno del otro, pero se condicionan y se complementarı recíprocamente. ${ }^{16}$ L. Siep, op. cit., p. 8-9. 
época... La lógica de Hegel no explica nada (pseudo)aristotélicamente, sino que es recordada como Sinngebung, o donación de sentido, cuando ya es demasiado tarde... ${ }^{17}$.

El problema entonces es cómo se concibe la relación del concepto y la representación en la filosofía ${ }^{18}$. Nosotros no podemos proceder como las ciencias empíricas, dice Hegel, mediante un simple procedimiento de

${ }^{17}$ F. Duque, op. cit., p. 66.

18 Angelica Nuzzo ha ensayado explicitar la forma de la articulación de los dos lenguajes con los que juega la exposición de Hegel en las partes reales de su filosofía, el lenguaje propio de los contenidos, que es el lenguaje de la representación (con el cual se introducen en la trama conceptual tanto contenidos de la experiencia o de la existencia natural inmediata, como contenidos históricos, y también contenidos teóricos no especulativos de las ciencias naturales y sociales), y el lenguaje de la Ciencia de la Lógica, y ha sostenido la tesis de la existencia de una suerte de metalógica, o lógica combinatoria de estos dos lenguajes. "Begriff und Vorstellung sind die Konstitutionsprinzipien (WdL II, 285) zweier spezifischer Logiken, die als die Logik der Wissenschaft der Logik und als die Logik der realphilosophischen Teile der System abgeleitet und entwickelt werden" ("Begriff und Vorstellung. Zwischen Logik und Realphilosohpie", en Hegel-Studien, Bd. 25, 1990, p. 46). En su investigación sobre la lógica de la filosofía real esta autora ha puesto de relieve la estructura circular de fundamentación recíproca de la Ciencia de la Lógica y la filosofia real. En su libro posterior: Rappreseniazione e concetto nella 'logica' della 'Filosofia del diritto' di Hegel, Napoli, Guida, 1990, desarrolla más extensamente su investigación: "L'ipotesi di lavoro qui tenteremo di verificare, la tesi generale della presente ricerca, è che il rapporto tra 'logico' e 'reale' non sia per Hegel un rapporto rigidamente ed univocamente deduttivo... Detto in altri termini, il problema della logica della filosofia del diritto, inquanto problema della ontologia dello spirito oggettivo, non è quello di dimostrare la possibilità della 'corrispondenza' (Entsprechung) tra logico e reale... Hegel risolve di fatto la difficoltà presentata delia forma reale ed oggettiva dello spirito, affidando lo svolgimento della struttura sistematico-concetuale della Filosofia del Diritto ad una 'logica combinatoria' di due linguaggi teorici asai diversi tra loro [Sprache der Vorstellung-Sprache des Begriffs], come tali sempre mantenuti, eppure al tempo stesso integrati a collaborare al medesimo disegno teorico di fundamentazione di una 'scienza filosofica del diritto' (pp. 14 y 19). Cfr. de la misma autora: "The Lenguage of Hegel's Speculative Philosophy", en Hegel and Language, edited by J. O' Neill Surber, Albany (NY), State New York University, 2006. 
generalización a partir de lo singular que se tiene en la representación, sino que tenemos que mostrar "como el concepto se determina a sí mismo", como las determinaciones se despliegan en tanto exigencias necesarias de la naturaleza de la cosa. "Nosotros tenemos que confrontar este concepto con la configuración de nuestra representación, y aquí podemos encontrar por cierto que puede haber muchas cosas que no concuerdan (da findet sich denn wobl, daß manches nicht paßt)". Esta diferencia de la representación con el concepto da lugar precisamente a la crítica filosófica. "Pero lo que es lo esencial en las configuraciones de la realidad tiene que corresponder también en nuestra representación a lo que determina el concepto"19. Sin embargo, la pauta del "concepto que se determina a sí mismo" no está tomada directamente de la lógica del puro concepto, sin tener en cuenta el contenido jurídico, moral y político, y el examen de los desajustes tendrá que ser al mismo tiempo un examen del contenido y de la forma lógica. Hay que tener en cuenta siempre que la relación de la Lógica y la Filosofía Real no es la de una jerarquía unilateral de saberes, sino una relación circular de interacción recíproca. En tal sentido adhiero a la interpretación de F. Duque cuando escribe: "La Ciencia de la Lógica es únicamente inteligible tras la comprensión de las partes reales del sistema, de las que genéticamente procede, aun cuando ontológicamente las preceda. En otras palabras: La Ciencia de la Lógica no es sino una abstracción del sistema operativo obrante en la Filosofía de la naturaleza y del espíritu"20.

\section{4.}

Quiero volver otra vez todavía a la definición del $\int 1$ de la Filosofía del Derecho para poner de relieve que su formulación da a entender que el objeto de esta ciencia no es simplemente la idea del derecho en su unidad de concepto y realidad, sino que se trata de estos dos momentos considerados también en su diferencia. Si esta interpretación es correcta, podemos decir que al primer momento, que es el desarrollo del concepto, se refieren las dos primeras partes, sobre el derecho abstracto y sobre la moralidad, o sobre el derecho del sujeto moral. El segundo momento de la idea es el que aborda

${ }^{19}$ Lección de Filosofia del Derecho 1823/24, loc. cit.

${ }^{20} \mathrm{~F}$. Duque, op. cit., p. 64. 
la Tercera Parte sobre la eticidad. Aquí se trata de la libertad o del derecho en cuanto realizado en el sistema de las instituciones. Pero las escuetas dos líneas del $\int 1$ dan a entender todavía algo más, por cuanto al referirse a este segundo momento no habla simplemente de la realidad (Wirklichkeit) del derecho, sino de "su realización (dessen Verwirklichung)". Esta expresión se puede interpretar como alusión al proceso del desarrollo del concepto, pero también al proceso de la realización de la libertad en la historia, es decir, como la realidad social e institucionalizada que la libertad se da a sí misma en la interacción de los individuos, a través del reconocimiento recíproco ${ }^{21}$. Este proceso culmina para Hegel en su época a través de la Revolución Francesa, en el moderno Estado de derecho. Esta es precisamente la materia de la última sección de la Tercera Parte sobre la historia del mundo ${ }^{22}$.

Puede decirse entonces que, en términos generales, la obra está compuesta esencialmente de dos grandes partes (las cuales no coinciden con las divisiones materiales del texto). Conforme a lo explicado, la segunda parte de esta ciencia, que es la parte sustancial, ( $y$ la primera in via inventionis, aunque el ordo expositionis siga la secuencia inversa), comienza con la eticidad, en el $\int 341$. Esta segunda parte (que estudia el mundo ético en su realidad institucional concreta y en su devenir, en cuanto contrapuesta a la primera que se mantiene en un nivel de análisis conceptual reconstructivo abstracto del derecho y la moralidad), comprende tanto la consideración sincrónica de la estructura real concreta del espíritu objetivo como la consicieración diacrónica del devenir de la conciencia de la libertad, a través de la Filosofía de la Historia.

21 "El puro pensamiento de la libertad es una mera abstracción y tiene que realizarse... Nosotros sostenemos, que la libertad en cuanto existente es el derecho" (VRph 3-171-172). El concepto de la libertad es existente en cuanto es reconocido, y esta existencia de la libertad reconocida es el derecho.

22 "En la determinación del objeto de la Filosofía del Derecho se expresa ya la doble perspectiva de la exposición y el papel complementario de la filosofía del derecho y la filosofía de la historia: la filosofía del derecho realiza el tratamiento del concepto del derecho y la configuración que deviene de él, la filosofía de la historia -como segunda parte de la ciencia filosófica del derecho- trata su realización histórica" (D. Brauer, Dialektik der Zeit. Untersuchungen $z^{n}$ Hegels Metaphysik der Weltgeschicht, Stuttgart, Frommann-Holzboog, 1982, p. 30). 
La primera edición de la obra publicada por el autor presenta una doble portada interior con dos títulos diferentes. En la primera el título se desdobla a su vez en dos: Naturrecht und Staatswissenschaft im Grundrisse (Derecho natural y ciencia del estado en compendio). En el centro de la página, y con caracteres de mayor tamaño se ubica la palabra Staatswissenschaft (Ciencia del Estado). Este doble título conecta dos conceptos pertenecientes a tradiciones de pensamiento que son diferentes y rivales $^{23}$. Resulta incluso una provocación desconcertante al conjugar el derecho natural con la política o ciencia del Estado. En la página siguiente el título es: Grundlinien der Philosophie des Rechts (Lineamientos fundamentales de Filosofía del Derecho). La diagramación y la tipografía resaltan en este caso la expresión: Filosofía del Derecho.

El doble título de la primera portada interior se puede comprender entonces como referido a las dos grandes partes de la obra. La primera parte, a la que se refiere la expresión "Derecho natural" (Naturrechit), comprende el derecho abstracto y la moralidad. (Hegel denomina también a la moralidad "el derecho de la persona particular" para diferenciarlo del derecho de la persona abstracta o derecho abstracto). La segunda parte, a la que alude la expresión "Ciencia de Estado" (Staaiswissenschafit) se refiere a la eticidad, cuyo capítulo principal trata del Estado. Esta comprensión del título abona también entonces la interpretación de que, no obstante la división material que presenta la obra en tres partes, formalmente puede hablarse de dos grandes partes, de las cuales la primera está referida sin embargo también a la segunda, como lo sugiere la misma tipografía del título en la edición original. En cuanto al título de la segunda portada: "Filosofía del Derecho", que es el que ha prevalecido, puede decirse que en la concepción de Hegel sintetiza los dos conceptos anteriores. En el lenguaje contemporáneo más corriente, en cambio, este título requiere ahora otra aclaración, porque con él se denomina habitualmente una disciplina especial de las ciencias jurídicas, y de hecho la filosofía del derecho ha pasado a ser después de Hegel una cosa de juristas más que de filósofos ${ }^{24}$. Quizás sea

${ }^{23}$ Cfr. M. Giusti, Hegels Kritik der Modernen Welt. Über die Auseinandersetzung mit den geschichtlichenund systematischen Grundlagen der praktischen Pbilosophie, Würzburg, Könighausen und Neumann, 1987.

${ }^{24}$ Cfr. W Hassemer, "Rechtsphiliphie, Rechtswissenschaft, Rechtspoiitik", en Archiv für Rechts und Sozialpobilosopbie, 44, 1991, pp. 130-143. 
preciso aclarar entonces que la Filosofia del Derecho de Hegel tiene poco que ver con lo que suele hacerse bajo este título en las Facultades de Derecho, y que su contenido se corresponde con el campo de lo que en la tradición clásica, que proviene de Aristóteles, se conocía como "Filosofía práctica", cuya disciplina fundamental era en el filósofo griego la Política. La obra de Hegel se ubica en esta tradición de pensamiento, pero la antigua Filosofía práctica se ha transformado en su Sistema en la Filosofia del espiritu objetivo, que es la denominación bajo la cual trata toda esta misma materia en la Enciclopedia de las Ciencias Filosóficas. Esta parte del sistema, publicada en un libro independiente con los títulos que acabo de reseñar, articula desde un punto de vista filosófico todo el contenido de las ciencias del espíritu en la denominación alemana de la época: la Ética, la Ciencia del Derecho, la Teoría social, la Economía, la Política, la Teoría del Estado y la Filosofía de la historia universal.

5.

Volviendo a los problemas más específicos del método y la estructura de la obra que habíamos planteado, quiero agregar ahora que en el tratamiento del derecho y de la moralidad, los cuales son primeros en el orden expositivo que va de lo abstracto a lo concreto, realiza Hegel al mismo tiempo, la exposición y la crítica (Darstellung und Kritik) de estos conceptos (que la moderna filosofía del derecho natural y de la moral han hecho objetos de la ciencia como figuras independientes) ${ }^{25}$, a fin de mostrar precisamente su carácter abstracto, y la imposibilidad de pensarlos como realidades en sí, o a fin de mostrar las contradicciones que entraña esta manera de pensar. Su exposición explicita la racionalidad interna propia de estos conceptos, pero pone de relieve al mismo tiempo las insuficiencias de los mismos en esta forma abstracta, teniendo en vista la totalidad concreta del mundo ético, en el cual cobran existencia, y a partir del cual han sido

25 "So hat die Idee, wie sie als Familie bestimmt ist, die Begriffsbestimmungen zur Voraussetzung, als deren Resultat sie in Folgenden dargestellt werden wird. Aber $\mathrm{da} ß$ diese inneren Voraussetzungen auch schon als Gestaltungen, als Eigenthum, Vertrag, Moralität pp vorhanden seien, dieß ist die andere Seite der Entwicklung, die nur in höher vollendeter Bildung es zu diesem eigenthümlich gestalteten Dasein ihrer Momente gebracht hat" (VRph, 1824/25, 160). 
abstraídos sus conceptos. Parece imposible comprender por lo tanto el pasaje del derecho abstracto a la moralidad, y de la moralidad a la eticidad como un desarrollo inmanente del concepto, que progresa lógicamente de lo abstracto a lo concreto, porque el final de las dos primeras partes demuestra más bien lo contrario, a saber, que su desarrollo inmanente no se sostiene ni produce por sí mismo ningún resultado verdadero y concreto. El demostrar esto es, además, expresamente uno de los objetivos declarados de la crítica que el propio autor lleva a cabo en esos capítulos contra la metodología del jusnaturalismo.

La interpretación esbozada concuerda en parte con la que ha desarrollado Michael Theunissen, quien marca una diferencia muy fuerte en la Filosofía del Derecho entre los textos crítico negativos de las concepciones jusnaturalistas y los textos que exponen el propio punto de vista afirmativo de Hegel:

La crítica la lleva a cabo él en la exposición del derecho abstracto y de la moralidad; al punto de vista afirmativo se refiere en la doctrina sobre la eticidad. La cuestión que se plantea entonces es la de la conexión entre el derecho abstracto y la moralidad por una parte, y la eticidad por otra parte... El movimiento que lleva del derecho abstracto, pasando por la moralidad, a la eticidad, en cuanto las dos esferas subordinadas son no verdaderas, no puede ser por lo tanto un desarrollo progresivo (Entwicklung) en el sentido de que el mismo "concepto" se fuera enriqueciendo... En cuanto el derecho abstracto se invierte al final en la injusticia y la moralidad cae en el mal, ambos se extravían, por así decirlo, o se pierden en ellos mismos... La génesis de la eticidad no tiene lugar por lo tanto en el ámbito de la moralidad, como tampoco esta última tiene su génesis en el campo del derecho ${ }^{26}$.

Este punto de vista no permite comprender el desarrollo de la materia a partir de la mera lógica del concepto. $\mathrm{H}$. Ottmann, al hacer un balance de

26 M. Theunissen, "Die verdrängte Intersubjektivität in Hegels Philosophie des Rechts", en Dieter Henrich u. Rolf-Peter Horstmann, op. cit., p. 336, 339 y 344. El autor intenta demostrar allí su tesis mediante un análisis del texto que nosotros no podemos reproducir aquí, especialmente de las transiciones de una esfera a otra y de la lógica subyacente a cada una de ellas. 
esta discusión, insinúa que es preciso reconocer "la función crítico propedéutica de las dos primeras esferas", y que se puede leer "el derecho abstracto y la moralidad como una suerte de Fenomenología de la conciencia jusnaturalista moderna"27. Al igual que en la Fenomenología del Espiritu, el filósofo muestra cómo cada una de estas figuras de la conciencia, siguiendo la lógica interna de su desarrollo, conduce a su propia autorefutación. Este es el sentido hegeliano de la "crítica interna"28.

Extremando estas interpretaciones de las dos primeras partes se llegaría a una fractura de la unidad de la obra, como un agregado de dos tratados que se complementan, pero que están concebidos de manera diferente y desarrollados con métodos distintos: un tratado crítico-negativo del derecho y de la moralidad, que prepara el camino para la exposición positiva de la doctrina propia de Hegel contenida en el tratado de la eticidad. Pero, contra una división demasiado fuerte y esquemática, hay que decir que la Tercera Parte une también la exposición con la crítica. La exposición se construye a lo largo de toda la obra en confrontación crítica con diferentes concepciones antiguas y modernas. La crítica se plantea desde la "Introducción" en dos frentes: 1) contra las abstracciones del derecho natural "revolucionario" y, 2) contra la doctrina de la resturación sostenida por la llamada escuela histórica del derecho ${ }^{29}$; pero se abre luego en otras ramificaciones, como la crítica de la moralidad kantiana en cuanto contrapuesta a la eticidad; la crítica de la eticidad inmediata y sustancialista.

27 A. Nuzzo ha renovado esta tesis de la existencia de una veta metódica. fenomenológica en la Filosofía del Derecho, limitándola y precisándola (Rappresentazione e concetto nella 'logica' della 'Filosofia del diritto' di Hegel, pp. 36-41), después de los intensos debates suscitados por la propuesta hermenéutica de K.-H. Ilting de comprender toda esta obra como "una fenomenología de la conciencia de la libertad".

${ }^{28}$ En las Lecciones de 1824/25 se toma precisamente al derecho de propiedad privada ("das Eigenthumsrecht, eine Weise des Begriffs sich zu realisieren, die ibm aber nicht angemessen ist") como ejemplo de este progreso dialéctico del concepto y de la voluntad, que niega las determinaciones que no son adecuadas a la naturaleza del concepto: "Diß ist denn die Dialektik, dieß Negiren eine: Beschränktheit, einer bestimmten Stufe, die damit sich nur als untergeordnet zeigt" (VRph, 1824/25, 158159.

${ }^{29}$ Cfr. J. De Zan, op cit., pp. 393-409. 
del concepto platónico del Estado que no puede hacer lugar al desarrollo de la particularidad independiente y a la diferenciación de la sociedad civil; la crítica de la teoría contractualista del origen de la sociedad y del derecho, etc.

Yo propondría por lo tanto una explicación parcialmente distinta para este mismo problema. Creo que no es posible comprender las dos primeras partes en clave puramente crítico-negativa, como pretende Theunissen, sino que ya en ellas se articulan la crítica y la exposición afirmativa de la propia concepción de Hegel, que hace suyos principios fundamentales del jusnaturalismo moderno y de la moralidad kantiana. Es en esta doble perspectiva que hay que leer especialmente la exposición hegeliana de estas dos primeras partes. Pero como la Tercera Parte une también la exposición del punto de vista positivo del autor con la crítica de concepciones antiguas y modernas, aunque esta última se refiere aquí a conceptos más puntuales, hay que decir que el tratamiento se elabora de manera consistente con el mismo método a lo largo de toda la obra.

6.

Derecho y moralidad como momentos de la eticidad concreta, tienen en lo tercero, en lo ético, su origen y fundamento. Pero en la exposición filosófica, nos dice Hegel, este concepto de la eticidad se tiene que demostrar y tiene que aparecer por lo tanto como resultado, a partir de aquellos momentos. Considerados en sí mismos los momentos del concepto son abstracciones. Y si bien lo abstracto se funda en lo concreto, y lo concreto es lo único verdaderamente real, la demostración del concepto tiene que comenzar sin embargo con lo abstracto para que la verdad se produzca como resultado de la exposición $(\$ 32)$. El desarrollo del texto cumple este objetivo, pero por un camino diferente al que podría esperarse, porque las configuraciones del derecho y de la moralidad, tal como se han diferenciado y autonomizado en la historia, no permiten el pasaje de un momento a otro como momentos de un desarrollo inmanente del concepto, según hemos visto, sino que lo obturan, y hacen imposible la dialéctica del concepto. Por eso el resultado del desarrollo de cada uno de estos momentos tiene que ser un resultado crítico negativo, que muestra más bien la necesidad de abandonar los puntos de vista precedentes para 
recuperar los conceptos del derecho y la moralidad como existentes recién al final, en la última parte, como momentos de la eticidad concreta.

El desarrollo de las configuraciones reales de la idea ética produce una secuencia histórica, mientras que la serie de las determinaciones abstractas del concepto sigue un orden lógico, y no temporal. 'Y así es como tenemos nosotros dos series, una serie de determinaciones conceptuales, y una serie de configuraciones de la existencia", por un lado: derecho abstracto, moralidad, eticidad; y por otro lado: familia, sociedad civil, Estado. El hecho de que "los caminos de la deducción científica y de los fenómenos del mundo (der erscheinenden Welt) sigan una dirección inversa", se explica porque el concepto, en cuanto es resultado, tiene que presuponer sus determinaciones abstractas. Las diferencias del orden de los conceptos en la ciencia y en la estructura ontológica de su objeto, que ya estaban señaladas en la Ciencia de la Lógica, se complica en la filosofía del espíritu, dónde es preciso tener en cuenta además el orden del tiempo. "Hay que señalar que los momentos, cuyo resultado es una forma ulterior más determinada [y concreta], son anteriores a ella en cuanto determinaciones conceptuales en el desarrollo que hace la ciencia de la idea, pero no son anteriores [a lo concreto] como configuraciones, en el desarrollo temporal" (Rph \$32 Obs,). Lo que puede confundir todavía al lector que tiene en cuenta estas distinciones es el hecho de que el orden de la exposición del texto de la Filosofía del Derecho comienza, en las dos primeras partes, con la secuencia de las determinaciones del concepto, mientras que en la tercera adopta el orden del desarrollo temporal.

Hegel mismo ha considerado como problemático el comienzo de su exposición sistemática de la Filosofía del Derecho, y se ha detenido a justificarlo en diferentes lugares, especialmente en la Lección de $1823 / 24^{30}$. Sus aclaraciones dejan sin embargo puntos oscuros y me parecen sólo en parte satisfactorias. Después de dar la explicación lógica de su plan, él admite todavía que "uno podría preguntarse no obstante ¿por qué no comenzamos por lo concreto?" Y reitera enseguida que, si solamente lo tercero, la eticidad es lo verdadero y efectivamente real (das Wabre, das Wirkliche, das erste Wirkliche) "tanto más justificada está la pregunta ¿por qué no queremos

$30 V R p h 3,1823 / 24$, p. 164-170, $₫ 3$ i -32 . En lo que sigue cito estas páginas de los apuntes sin más referencias. 
comenzar con lo verdadero?”. La reiteración de estas preguntas se puede interpretar como expresión de su propia insatisfacción con el plan de la obra, y también como expresiones retóricas para evitar interpretaciones equivocadas.

El resultado negativo de la exposición de las dos primeras partes no debería sin embargo sorprender ni decepcionar al lector, como supone $\mathrm{H}$. Ottmann, quien describe de esta manera la experiencia de lectura de estas partes de la obra:

Cuanto más dispuesto se está a creer en las palabras de la "Introducción", que la exposición de la Filosofía del Derecho habrá de seguir el camino acostumbrado del progreso inmanente de lo abstracto a lo concreto $(\$ 30)$, tanto más confundido deja uno el libro después de la lectura. En lugar del progresivo ascenso desde lo abstracto hacia conceptos cada vez más ricos y concretos, lo que encuentra el lector es el ritmo de una secuencia de saltos (einer Springprozession) que, a un paso hacia adelante hace seguir enseguida otro paso atrás ${ }^{31}$.

Me parece en cambio que, si se cumplieran las expectativas de lectura de $H$. Ottmann, entonces sí nos encontraríamos con un resultado sorprendente y decepcionante, porque con ello quedaría refutada toda la concepción hegeliana del mundo ético, y quedaría corroborada la verdad de las teorías de Locke y de Kant.

El mismo tipo de problema que se plantea desde un punto de vista lógico en el pasaje de la Primera a la Segunda Parte y de la Segunda a la Tercera, se plantea también especialmente dentro de está última, en el pasaje del capítulo sobre "La sociedad civil" al capítulo sobre "El Estado", y de este al último apartado sobre "La historia universal". Quiero referirme para terminar a este último pasaje.

7.

En la Filosofía del Derecho y de la historia del mundo de Hegel, el concepto de la "Weltgeschichte" viene a ocupar el lugar que tenía en la.

${ }^{31}$ H. Ottmann, op. cit., p. 382. 
Filosofía del derecho de Kant el "Weltbürgerrecht", o Derecho cosmopolita. La estructura de la Filosofía del Derecho de Hegel presenta un desarrollo en cierto modo accidentado, como se ha visto, pero que (más allá de sus quiebres) sigue una línea claramente ascendente que culmina con la eticidad concreta de lo político, pero después del Derecho político interno del Estado, al tratar de la soberanía exterior y del Derecho político externo (das $V$ ölkerrecht), esta línea ascendente se quiebra nuevamente, no cierra sino que se abre y se produce en cierto modo una caída en la historia del mundo en la que los Estados particulares quedan sometidos a un acontecer imprevisible, se ven enfrentados en situaciones de guerra, y están destinados finalmente a perecer cuando su tiempo se ha cumplido, dando lugar al surgimiento de siempre nuevas formaciones históricas. El Derecho político externo, o Derecho internacional, es definido en términos que representan un paso atrás, al nivel del derecho abstracto, o de la moralidad, en cuanto tiene la forma del deber ser (die Form des Sollens), porque su realidad efectiva depende de diferentes voluntades soberanas que pueden respetar, o no, el imperativo moral kantiano: "no debe haber guerras", y hacer prevalecer sus intereses, o motivos políticos (\$331). "El principio del derecho internacional en cuanto derecho de lo universal debe ser un derecho reconocido como válido entre los Estados... y los tratados sobre los que se basan las relaciones mutuas entre los Estados deben ser respetados ( $\$ 333$ ), pero no hay ninguna garantía de que así suceda.

En la Enciclopedia Hegel se explaya un poco más sobre el pasaje del Estado a la historia universal. Quiero citar sin más comentario algunos párrafos de Encicl. \$S 545 a 548.

El Estado tiene finalmente la unilateralidad de ser la realidad inmediata de un pueblo singular, naturalmente determinado. En cuanto individuo singular es excluyente frente a otros individuos de esta misma clase. En las relaciones entre ellos tiene lugar el arbitrio y la contingencia, porque debido a la totalidad autónoma de estas personas [jurídicas] lo universal del derecho entre ellos es algo que debe ser, pero no es efectivamente real. Esta independencia puede llevar a los conflictos entre los Estados hasta una relación de fuerza, o un estado de guerra... En el estado de guerra la autonomía de los Estados queda puesta en juego, solamente se consigue mediante el reconocimiento mutuo de la libre individualidad de los pueblos, y se sostiene mediante tratados de paz que deben durar de manera 
perpetua... El espíritu determinado de un pueblo... está en el tiempo: ...tiene una historia interna. En cuanto espíritu limitado su autosuficiencia es de menor rango, y pasa a la historia general del mundo, cuyo acontecer expone la dialéctica de los espíritus particulares de los pueblos.

La historia del mundo no vuelve a alcanzar ya el nivel de una eticidad universal concreta, e institucionalizada como el estado de derecho interno. Y de hecho, observa Hegel, el impulso que abre los Estados hacia el exterior y genera todo tipo de relaciones entre los pueblos, no proviene del derecho, ni del Estado, sino de la sociedad civil (Rph $\left.\int 324\right)^{32}$, o de los principios universales de la moralidad y de las más elevadas realizaciones de la cultura, que trascienden el particularismo de los espíritus de los pueblos (lo que Hegel llama el espiritu absoluto) ${ }^{33}$. No obstante "el resto del estado de naturaleza", que no puede suprimirse en la sociedad civil $(\$ 200)$, las instituciones del mundo ético reemplazan aquella situación primitiva de no reconocimiento del otro y de sus derecho,

pero al final reaparece no solamente un resto, sino el propio estado de naturaleza sin más en las relaciones entre los Estados... La historia ha comenzado para Hegel como una lucha por el reconocimiento en el estado de naturaleza, pero en tanto subsiste la posibilidad de que los pueblos y los Estados se nieguen el reconocimiento recíproco, la historia seguirá siendo siempre una lucha por el reconocimiento ${ }^{34}$.

Este final puede parecer decepcionante porque no presenta un cierre mediante la universalización del derecho, ni promete la institucionalización de un universalismo político más allá de la pluralidad de los Estados particulares. El espíritu del mundo (Weltgeist) no se objetiva ya en cuanto tal, sino que permanece como una idea carente en sí misma de realidad (es

${ }^{32}$ Cfr. J. De Zan, op. cit., "Epilogo".

33 "Pero el espíritu pensante de la historia universal, en cuanto ha borrado al misrno tiempo aquellas limitaciones de los espíritus particulares de los pueblos y de su propia mundanidad, el que comprende su universalidad concreta y se eleva hasta el saber del espiritu absoluto..." (Encicl. \$552).

${ }^{34}$ Cfr. H. Ottmann, "Die Weltgeschichte", en L. Siep, op. cit., p. 286. 
decir, no como una "idea" en el sentido hegeliano). Los espíritus particulares de los pueblos encarnan diferentes principios del espíritu universal (\$345), pero el Volesgeist existe "como una pluralidad de elementos independientes, de tal manera que a cada pueblo corresponde uno de ellos" (\$ 346). En el contexto de la contingencia de la historia queda abierta la posibilidad, que parece inevitable, de la guerra. "En las guerras y rivalidades que surgen en estas circunstancias, el momento de la lucha por el reconocimiento en referencia a un determinado contenido, constituye el rasgo por el que adquieren una significación para la historia universal" ( $\mathbb{\Omega}$ 351). Este final puede interpretarse como expresión del realismo político del filósofo, el cual es más fuerte que su voluntad de sistema, pero también de su valoración de la individualidad de los pueblos, de las diferencias históricas de las culturas y las formas de vida (\$346), que hay que preservar y reafirmar independientemente de las pretensiones de validez universal del pensamiento, del universalismo de la sociedad civil, y de la globalización económica de los mercados, como diríamos hoy, que Hegel veía ya en su época como una consecuencia lógica del desarrollo del capitalismo.

\section{Resumen}

El comienzo de la Filosofía del Derecho con el concepto de "Derecho abstracto" y la "moralidad" presenta la apariencia de una forma de tratamiento típica del individualismo metodológico de las teorías jusnaturalistas. Hegel aclara sin embargo que el plan de la exposición no refleja ni el orden genético, o temporai, ni la estructura ontológica de la cosa. La pregunta que tratará de responder este artículo es, entonces, a qué criterios responde esta problemática estructura del plan de la obra. La tesis que sostiene el autor es que las dos primeras partes de la obra se tienen que leer principalmente como una exposición crítico-negativo de las teorías vigentes, y que recién la tercera parte sobre las instituciones de la eticidad expone positivamente el pensamiento de Hegel. Pero el tratamiento del derecho y la moralidad no es tampoco meramente negativo, sino que retoma y hace suyos al mismo tiempo principios fundamentales del jusnaturalismo y de Kant, como condiciones necesarias para comprensión de las instituciones de la sociedad y del Estado modernos.

Palabras clave: Derecho abstracto, moralidad, jusnaturalismo, eticidad. 


\begin{abstract}
The beginning of Pbilosopby of Right with the concept of "Abstract Right" and "morality" presents the appearance of a form of treatment typical of the methodological individualism of natural right theories. Nevertheless, Hegel makes it clear that the expository plan does not reflect the genetic, temporal or ontological structure of the issue. This article will thus try to give an answer to the question about the criteria to which this problematic structure of the work plan responds. The thesis sustained by the author is that the first two parts of the work have to be read mainly as a critical-negative exposition of the current theories, and only the third part about the institutions of Sittlichleit provides a positive exposition of Hegel's thought. The treatment of Right and morality is not merely negative either, but takes up again and at the same time conforms to Kant's and Natural Rights fundamental principles, as necessary conditions for the comprehension of the institutions of modern society and modern State.
\end{abstract}

Key words. Abstract Right, morality, natural right theories, Sittlicbkeit. 